\title{
Do magnetospheric shear Alfvén waves generate sufficient electron energy flux to power the aurora?
}

Article

Published Version

Original article

Watt, C. E. J. and Rankin, R. (2010) Do magnetospheric shear Alfvén waves generate sufficient electron energy flux to power the aurora? Journal of Geophysical Research, 115. A07224. ISSN 0148-0227 doi: https://doi.org/10.1029/2009JA015185 Available at https://centaur.reading.ac.uk/32811/

It is advisable to refer to the publisher's version if you intend to cite from the work. See Guidance on citing.

Published version at: http://dx.doi.org/10.1029/2009JA015185

To link to this article DOI: http://dx.doi.org/10.1029/2009JA015185

Publisher: American Geophysical Union

All outputs in CentAUR are protected by Intellectual Property Rights law, including copyright law. Copyright and IPR is retained by the creators or other copyright holders. Terms and conditions for use of this material are defined in the End User Agreement.

$\underline{\text { www.reading.ac.uk/centaur }}$ 
Central Archive at the University of Reading

Reading's research outputs online 


\title{
Do magnetospheric shear Alfvén waves generate sufficient electron energy flux to power the aurora?
}

\author{
C. E. J. Watt ${ }^{1}$ and R. Rankin ${ }^{1}$ \\ Received 9 December 2009; revised 26 January 2010; accepted 4 March 2010; published 27 July 2010.
}

[1] Using a self-consistent drift-kinetic simulation code, we investigate whether electron acceleration owing to shear Alfvén waves in the plasma sheet boundary layer is sufficient to cause auroral brightening in the ionosphere. The free parameters used in the simulation code are guided by in situ observations of wave and plasma parameters in the magnetosphere at distances $>4 R_{E}$ from the Earth. For the perpendicular wavelength used in the study, which maps to $\sim 4 \mathrm{~km}$ at $110 \mathrm{~km}$ altitude, there is a clear amplitude threshold which determines whether magnetospheric shear Alfvén waves above the classical auroral acceleration region can excite sufficient electrons to create the aurora. Previous studies reported wave amplitudes that easily exceed this threshold; hence, the results reported in this paper demonstrate that auroral acceleration owing to shear Alfvén waves can occur in the magnetosphere at distances $>4 R_{E}$ from the Earth.

Citation: Watt, C. E. J., and R. Rankin (2010), Do magnetospheric shear Alfvén waves generate sufficient electron energy flux to power the aurora?, J. Geophys. Res., 115, A07224, doi:10.1029/2009JA015185.

\section{Introduction}

[2] Observational evidence supports the idea that shear Alfvén waves (SAWs) are involved in auroral electron acceleration. Polar spacecraft observations at $4-6 R_{E}$ radial distance [Wygant et al., 2000; Keiling et al., 2002, 2003], and Cluster observations at $\sim 5 R_{E}$ radial distance [Vaivads et al., 2003] demonstrate that the Poynting flux contained in shear Alfvén waves is sufficient to account for auroral brightening at locations magnetically conjugate to the satellites. Other statistical analyses of Polar spacecraft data [Janhunen et al., 2006] showed that the Alfvén wave amplitude decreases significantly at 4-5 $R_{E}$ radial distance, suggesting that the waves transfer their energy to provide parallel electron acceleration at this location. Accelerated populations of electrons are often observed accompanying SAWs in the plasma sheet or plasma sheet boundary layer [Angelopoulos et al., 2002; Wygant et al., 2002; Morooka et al., 2004], further strengthening the connection between shear Alfvén waves and electron acceleration processes.

[3] Even more compelling evidence for the link between shear Alfvén waves and auroral electrons can be gained from observations at lower altitudes. The Fast Auroral Snapshot (FAST) satellite has provided much insight into the different forms of accelerated electrons and their association with SAWs [e.g., Chaston et al., 2000, 2002]. Comparisons between wave and particle measurements at Cluster and FAST [Chaston et al., 2005], and Polar and FAST [Dombeck et al., 2005], when the spacecraft are magnetically conjugate

\footnotetext{
${ }^{1}$ Department of Physics, University of Alberta, Edmonton, Alberta, Canada.

Copyright 2010 by the American Geophysical Union. 0148-0227/10/2009JA015185
}

to one another, are consistent with the idea that SAWs can accelerate electrons downward in the field-aligned direction. Ergun et al. [2005] presented FAST observations of largeamplitude SAWs that were accompanied by accelerated electrons and large-amplitude parallel electric fields at altitudes of 2000-3000 km. Statistical analysis of the occurrence of SAWs with short perpendicular scale lengths, along with the occurrence of accelerated electron signatures, shows that dispersive Alfvén waves can provide a significant, though not dominant, mechanism for accelerating electrons above auroral regions [Chaston et al., 2007]. The low-altitude Freja satellite also provided evidence for electron acceleration by SAWs, showing that the acceleration mechanism must provide acceleration above the spacecraft as well as local acceleration [Andersson et al., 2002a, 2002b].

[4] Comparisons between in situ electron data from lowaltitude orbiting spacecraft and auroral imagers on aircraft [Stenbaek-Nielsen et al., 1998] or on the satellite itself [Asamura et al., 2009] show that it is possible to relate specific auroral arc structures with electron acceleration events. High-energy inverted-V structures in electron energy spectra are shown to be associated with discrete arcs, although less energetic energy-dispersed electrons are also observed in conjunction with auroral displays and may contribute to weaker aurora. Semeter et al. [2005] performed a detailed comparison between ground-based optical and radar measurements with electron precipitation from the FAST spacecraft, and they concluded that the electron spectra, measured in regions of intense Alfvén wave activity, can account for the auroral characteristics observed on the ground.

[5] Rocket measurements between 300 and $1000 \mathrm{~km}$ altitude also provide evidence for the association between SAWs and auroral forms. Large-amplitude SAWs are observed in many cases in tandem with field-aligned electron acceleration 
and enhanced electron energy flux [Boehm et al., 1990; Ivchenko et al., 1999; Lynch et al., 1999; Hallinan et al., 2001]. Ground-based optical measurements of auroral arcs are shown to be consistent with acceleration by dispersive Alfvén waves [Semeter and Blixt, 2006; Semeter et al., 2008].

[6] It was suggested that electron acceleration caused by shear Alfvén waves can provide an essential link between events in the geomagnetic tail and the ionosphere (e.g., magnetic reconnection and substorm expansion phase onset [Angelopoulos et al., 2008; Østgaard et al., 2009; Rae et al., 2009]). However, the interaction between SAWs and electrons in the warm plasma of the magnetotail is not well understood, and most previous studies have concentrated on regions of the magnetosphere that are closer to the ionosphere.

[7] The aforementioned observations cluster around three locations in near-Earth space: 4-7 $R_{E}$ radial distance (Polar, Cluster, etc.), 300-4200 km altitude (FAST, Freja, rockets), and indirect measurements from the ground of the auroral emission region between $\sim 100$ and $300 \mathrm{~km}$ altitude. The details of the physical mechanism that connects all of these observations is not known. It is clear from the observations that the energy and number of accelerated electrons can vary dramatically from one event to another. We require models to predict which wave characteristics are important for electron acceleration and which plasma conditions are conducive to wave-particle interactions.

[8] The most straightforward way to study electron acceleration in SAWs is to use a modified fluid code to predict the strength of the parallel electric field using linear kinetic predictions [e.g., Rankin et al., 1999a, 1999b; Rankin and Tikhonchuk, 1998; Streltsov and Lotko, 1999]. Details of the electron acceleration mechanism can then be studied by following test particles as they are influenced by the parallel electric field force and mirror force [Chaston et al., 2000, 2002, 2003a, 2003b; Thompson and Lysak, 1996; Kletzing, 1994; Kletzing and Hu, 2001; Su et al., 2004, 2007]. However, these models lack self-consistency, and agreement with observations can sometimes only be obtained through application of specfic plasma conditions which may prove too restrictive in Earth's magnetosphere.

[9] More complicated models of shear Alfvén waves and electron acceleration in the magnetosphere accent the kinetic and nonlocal nature of the wave-particle interaction between shear Alfvén waves and electrons [Tikhonchuk and Rankin, 2000, 2002; Lysak and Song, 2003a, 2003b, 2005], although these models are linear and so cannot include the full nonlinear dynamics that has been shown to be important.

[10] Self-consistent simulations of SAWs have demonstrated that nonlinear wave evolution is a key part of the SAW-electron acceleration process [Clark and Seyler, 1999; Damiano et al., 2003, 2007; Damiano and Wright, 2005; Genot et al., 2000, 2001, 2004; Watt et al., 2004, 2005, 2006; Tsiklauri et al., 2005; Seyler and Liu, 2007; Swift, 2007; Watt and Rankin, 2009], although none of these studies make a quantitative prediction of whether the energy and flux of the accelerated electrons predicted by the models would be sufficient to cause auroral brightening. In this paper, we use the self-consistent DK-1D model [Watt and Rankin, 2008a] to investigate the energy and energy flux of electrons accelerated by SAWs and test whether the resulting flux can be responsible for auroral displays. Chaston et al. [2003b] extensively studied the possible auroral consequences of electron acceleration in the cold plasma region close to the Earth. We focus instead on the region of warm plasma in near-Earth space in the plasma sheet boundary layer at $\sim 4-7 R_{E}$ radial distance. Where possible, we use published observations to guide our choice of wave parameters in the model. Section 2 describes the model in detail and section 3 provides the results from simulations designed to study the shear Alfvén wave-electron interaction in the warm magnetosphere. Section 4 contains a discussion of our results in the context of the observational evidence that demonstrates an association between SAWs and auroral electron acceleration. Our conclusions are presented in section 5 .

\section{DK-1D Simulation}

[11] The DK-1D code [Watt and Rankin, 2008a] is a numerical simulation code which follows the self-consistent kinetic electron interaction with the electromagnetic fields of a shear Alfvén wave. The three governing equations [Watt et al., 2004] are the electron drift-kinetic equation

$$
\begin{aligned}
\frac{\partial f}{\partial t} & +\left(p_{\|}-\frac{q_{e}}{m_{e}} A_{\|}\right) \frac{\partial f}{\partial z}+\left\{\frac{q_{e}}{m_{e}}\left[\left(p_{\|}-\frac{q_{e}}{m_{e}} A_{\|}\right) \frac{\partial A_{\|}}{\partial z}-\frac{\partial \phi}{\partial z}\right]\right. \\
- & \left.\frac{\mu}{m_{e}} \frac{\partial B_{0}}{\partial z}\right\} \frac{\partial f}{\partial p_{\|}}=0
\end{aligned}
$$

Ampère's law

$$
A_{\|}=\frac{\mu_{0} q_{e} F}{k_{\perp}^{2}+\mu_{0} \frac{q_{e}^{2}}{m_{e}} Z},
$$

and an equation for the scalar potential which is derived from the ion polarization equation

$$
\frac{\partial \phi}{\partial t}=-\frac{v_{A}^{2}}{k_{\perp}^{2}} \frac{\partial\left(k_{\perp}^{2} A_{\|}\right)}{\partial z} .
$$

In these equations, $f\left(z, p_{\|}, \mu, t\right)$ is the electron distribution function and is a function of distance along the field line $z$, the parallel canonical momentum per unit mass $p_{\|}=v_{\|}+$ $\left(q_{e} / m_{e}\right) A_{\|}$, the magnetic moment $\mu=m_{e} v_{\perp}^{2} /\left(2 B_{0}\right)$, and the time $t ; \phi(z, t)$ and $A_{\|}(z, t)$ are the scalar and parallel vector potentials, respectively; and the zero and first moments of the distribution function are given by

$$
Z=\int_{0}^{\mu_{M}} \int_{-p_{M}}^{p_{M}} \frac{B_{0}}{m_{e}} f d p_{\|} d \mu
$$

and

$$
F=\int_{0}^{\mu_{M}} \int_{-p_{M}}^{p_{M}} p_{\|} \frac{B_{0}}{m_{e}} f d p_{\|} d \mu,
$$

where $\mu_{M}$ is the maximum value of $\mu$ and $p_{M}$ is the maximum value of $p_{\|}$used in the simulation. These maximum values are chosen such that the magnitude of $f\left(z, \pm p_{M}, \mu, t\right)$ or $f\left(z, p_{\|}, \mu_{M}, t\right)$ is always small, regardless of the size of the local electron drift velocity or vector potential.

[12] The initial distribution function $f(t=0)$ is an isotropic Lorentzian distribution function in both parallel and perpendicular velocity with $\kappa=5$ [Summers and Thorne, 1992]. Appropriate coordinate transforms are made to obtain $f\left(p_{\|}, \mu\right)$. 


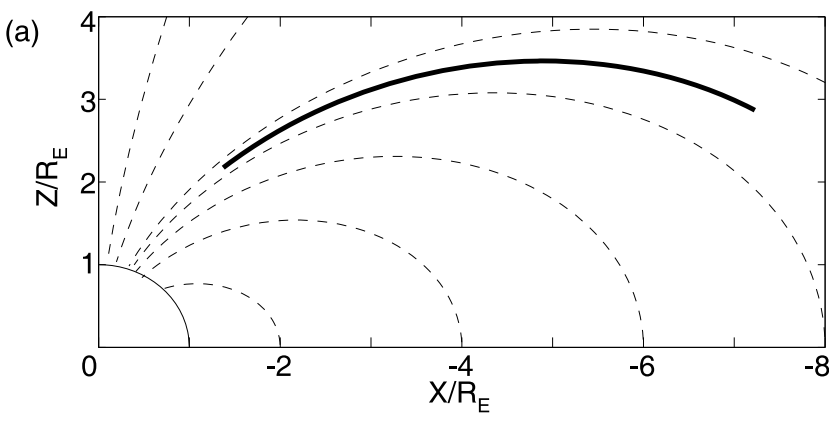

(b)

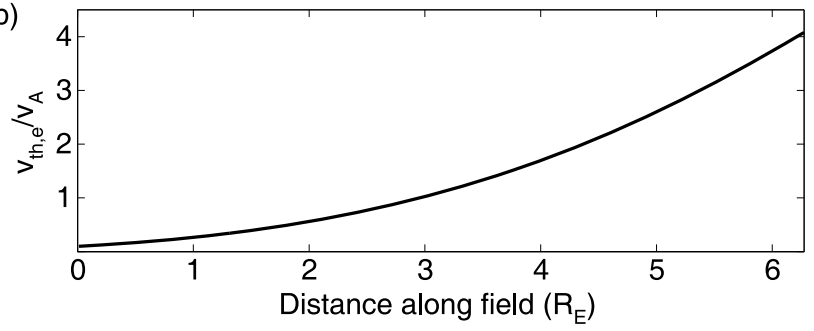

Figure 1. (a) Schematic of the simulation domain in an idealized dipolar magnetosphere. Simulation domain lies along the $L=9$ field line. (b) Variation of $v_{t h, e} / v_{A}$ throughout the simulation domain as a function of distance along the field line.

Observations indicate that electron distribution functions in the auroral source region and along geomagnetic field lines above the auroral oval often have high-energy tails that can be modeled by a Lorentzian $(\kappa)$ distribution with $\kappa<7$ [Christon et al., 1988; Olsson and Janhunen, 1998; Kletzing et al., 2003]; hence, we choose this model over the more common Maxwellian electron model [Damiano and Wright, 2005; Swift, 2007]. Previous simulation work [Watt and Rankin, 2002] showed that electron acceleration is affected by the form of the distribution function, so it is important to choose the more realistic Lorentzian model. Note that equation (1) gives $\partial f / \partial t=0$ for a Lorentzian distribution function in a dipolar magnetic field. The initial distribution function we chose is therefore a stationary solution of equation (1). Numerically, however, the evaluation of equation (1) involves adding two terms that are analytically the same size and of opposite sign. In most parts of the phase-space grid, we get an initial numerical evaluation of equation (1) that is not equal to zero, although it is very small. In these places, we make a numerical correction such that, for the initial equilibrium, $\partial f / \partial t=0$ everywhere in phase space.

[13] The drift-kinetic equation is integrated forward in time using the corner transport upwind method as described by [Leveque, 2002]. A van Leer gradient limiter [van Leer, 1974] is also applied to minimize numerical dissipation and dispersion. The moments calculated for equation (2) are obtained using a combination of a cubic spline integration method and the in-pairs method [see Horne and Freeman, 2001]. The electron distribution function and the vector potential are calculated at the same physical time $t=n \Delta t$, where $n$ is an integer. The scalar potential is calculated using a leapfrog method in time and space [e.g., Thompson and Lysak, 1996] at times $(n+1 / 2) \Delta t$.

[14] The ambient magnetic field $B_{0}$ is modeled using a dipolar field line with $L=9$ (see Figure 1a for a schematic diagram of the simulation domain). This magnetic field model was used because it is straightforward and $L=9$ is a reasonable choice for a magnetic field line that threads the plasma sheet boundary layer. We will investigate the effects of more realistic magnetic field geometries in future work. The electron number density and temperature are initially constant throughout the simulation domain with $n_{e}(t=0)=$ $10^{6} \mathrm{~m}^{-3}$ and $T_{e}(t=0)=200 \mathrm{eV}$. The values of number density and temperature are reasonable for auroral field lines, although observed electron temperatures can vary from hundreds of eV [Baumjohann, 1993; Angelopoulos et al., 2002; Janhunen et al., 2006] to a few keV [Wygant et al., 2002]. Future studies will focus on the dependence of this acceleration process on the temperature of the electrons. For this study, a value of electron temperature at the lower end of the observed range is selected to keep the runtime of the simulation short.

[15] The perpendicular wave number $k_{\perp}$ is scaled along the simulation domain according to the dipolar configuration of the magnetic field [Lysak and Lotko, 1996] with the perpendicular wave number $\lambda_{\perp}$ scaled to $4 \mathrm{~km}$ at an altitude of $110 \mathrm{~km}$. This choice of perpendicular wave number is consistent with the perpendicular wave numbers deduced by Wygant et al. [2002] in their study of Polar field observations in the plasma sheet boundary layer. Figure $1 \mathrm{~b}$ shows the variation of $v_{t h, e} / v_{A}$ along the field line (coordinate $z$ ) for the simulations used in this study.

[16] The results from four simulations are compared in section 3. SAWs are excited by adding the waveform $\phi_{w}=$ $\phi_{0} \exp \left[-(t-3 T)^{2} / 4 T^{2}\right] \sin (\omega t)$ to the scalar potential at the upper boundary, with a period $T=2 \mathrm{~s}$ and amplitudes $\phi_{0}=$ $100,400,800$, and $1600 \mathrm{~V}$ (the different simulations are labeled A, B, C, and D, respectively). Observations by Wygant et al. [2002] demonstrate that higher-frequency shear Alfvén waves with frequencies $<10 \mathrm{~Hz}$ may have small perpendicular scale lengths, although wave frequencies measured in situ in the magnetosphere by moving satellites are often subjected to the Doppler effect. Note that this choice of wave period ensures that the wave frequency is always below the ion cyclotron frequency $\Omega_{i}$, although the ratio $\omega / \Omega_{i}$ approaches 0.5 at the upper boundary of the simulation. As the wave enters the simulation domain, there is a transient period corresponding to the time it takes to establish a trapped electron population in the wave. The wave-particle interactions that occur subsequent to this injection are in the plasma regime where $\omega / \Omega_{i}$ is decreasing as the wave propagates earthward.

[17] In each simulation, the wave packet propagates in the $-z$ direction, interacting with the electrons as it travels toward the lower boundary of the simulation. At this lower boundary, the boundary conditions are such that $\partial A_{\|} / \partial z=0$, and the incoming distribution function has fixed $n_{e}, T_{e}$, and a drift velocity consistent with the parallel current required to support the wave vector potential. These boundary conditions result in partial reflection of the wave at the lower boundary.

[18] Note that the lower boundary is fixed at a point which represents $r=2.6 R_{E}$ radial distance in the physical magnetosphere. This allows for a reasonable size and resolution of the velocity space grid $\left(p_{\|}, \mu\right)$. If we had chosen a boundary for the simulation closer to the Earth, then the resolution required in $\mu$ space would be prohibitively large, leading to an unfeasibly long runtime for these simulations. We also chose 


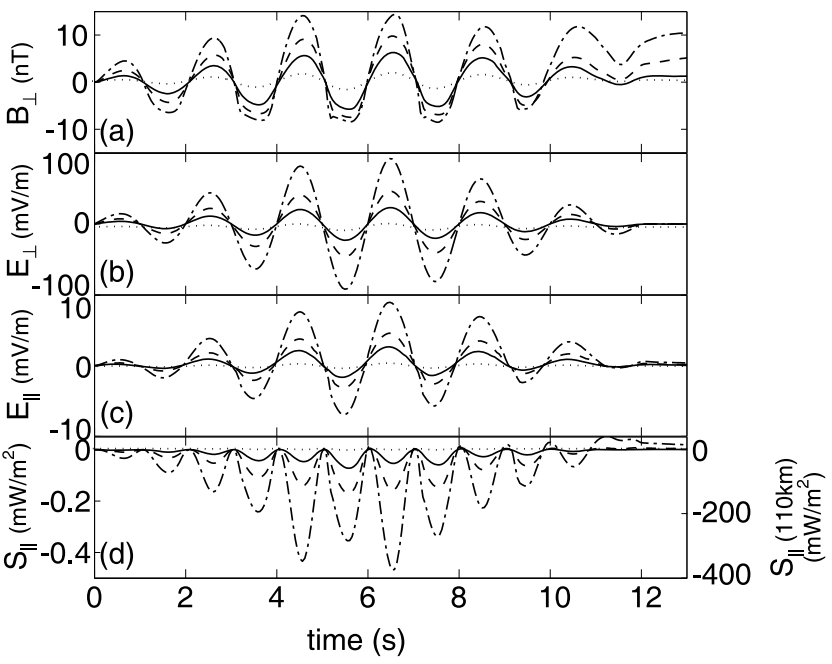

Figure 2. (a) Perpendicular magnetic field perturbation $B_{\perp}$, (b) perpendicular electric field perturbation $E_{\perp}$, (c) parallel electric field $E_{\|}$, and (d) parallel component of the Poynting vector $S_{\|}$measured in each simulation at $z=6.3 R_{E}(r \sim$ $7.8 R_{E}$ radial distance). The magnitude of $S_{\|}$is shown at $z=$ $6.3 R_{E}$ (left-hand axis) and mapped to a reference altitude of $110 \mathrm{~km}$ (right-hand axis). Dotted, solid, dashed, and dotdashed lines represent values from simulations A, B, C, and $\mathrm{D}$, respectively.

the location of the simulation domain (Figure 1a) such that our assumption of initially uniform number density and temperature is reasonable. Below the simulation lower boundary, the number density increases and the temperature decreases with decreasing altitude [Kletzing et al., 2003]. The physics of the low-altitude magnetosphere presents many challenges to modeling [see, e.g., Sydorenko et al., 2008], and so we investigate the behavior of SAWs at smaller radial distance in future work.

\section{Results}

[19] Figure 2 shows the evolution of the wave magnetic and electric fields at $z=6.3 R_{E}\left(r=7.8 R_{E}\right)$ for all three simulations (dotted, solid, dashed, and dot-dashed lines represent simulations A, B, C, and D, respectively). The wavefields have not yet been damped or altered through interaction with the electrons and so Figure 2 exhibits the initial profile of the waves before they interact with the electrons. The perpendicular magnetic field (Figure 2a) has a magnitude of $\sim 2 \mathrm{nT}$ for simulation $\mathrm{A}, \sim 5 \mathrm{nT}$ for simulation $\mathrm{B}, \sim 10 \mathrm{nT}$ for simulation $\mathrm{C}$, and $\sim 15 \mathrm{nT}$ for the most strongly driven simulation, D. The perpendicular electric (Figure $2 \mathrm{~b}$ ) fields have amplitudes $E_{\perp} \sim 6, \sim 20, \sim 45$, and $\sim 95 \mathrm{mV} / \mathrm{m}$ for simulation A, B, C, and $\mathrm{D}$. The parallel electric field (Figure $2 \mathrm{c}$ ) is generated because of the small perpendicular wavelength $k_{\perp} \lambda_{e} v_{t h, e} / v_{A}=$ 1.2 of the wave as it travels through the warm plasma [Goertz and Boswell, 1979; Nakamura, 2000]. Figure 2d shows the parallel component of the Poynting vector, calculated from the simulation wavefields. Negative values of $S_{\|}$indicate electromagnetic wave energy flux directed downward, toward the lower boundary of the simulation. For comparison with in situ observations of the magnitude of the Poynting vector [e.g., Wygant et al., 2000, 2002; Angelopoulos et al., 2002; Vaivads et al., 2003; Dombeck et al., 2005; Keiling, 2009], we also show the magnitude of $S_{\|}$mapped to a reference altitude of $110 \mathrm{~km}$ on the right-hand axis of Figure 2. The magnitudes of the waves and Poynting flux are reasonable for radial distances $>4 R_{E}$ in the plasma sheet boundary layer [Keiling, 2009], although the initial wave magnitudes in simulation $\mathrm{D}$ are slightly larger than most observations. We discuss the relationship between the strength of the waves driving these simulations and the observations in section 4 .

[20] Note that the negative perturbations in $B_{\perp}$ are not as large as the positive perturbations for simulations $\mathrm{C}$ and $\mathrm{D}$. This is because of the distortion of the distribution function as the wave travels through the plasma and is discussed in association with Figure 3. However, this distortion is different from the distortion of the distribution function into triangular shapes seen in plasma with $v_{t h, e} \ll v_{A}$ [Watt et al., 2004, 2005].

[21] Figure 3 demonstrates the different interactions between the wave and the electrons as the wave travels through the plasma for each of the four simulations. Snapshots of the electron distribution function are shown for $\mu=0$ and $t \sim 7.8 \mathrm{~s}$, concentrating on the upper region of the simulation domain where $v_{t h, e} \ll v_{A}$. As discussed by Watt and Rankin [2009], the electrons are trapped in the shear Alfvén wave near the top of the simulation domain, where plasma conditions for support of the parallel electric field are optimal. As the wave moves into plasma with a lower value of $v_{t h, e} / v_{A}$, the parallel electric field diminishes and the electrons escape the influence of the wave to form energy-dispersed beams. All simulations show a similar evolution, except for the width of the trapping islands in velocity space. As the initial wave amplitude increases, the trapping islands become wider, which is to be expected because the initial parallel electric field near the top of the simulation domain increases with wave amplitude.

[22] The resulting electron acceleration is shown in Figure 4 . Each part shows the differential electron energy flux $J_{E}(E, t)$ of downward electrons $\left(\mu=0, p_{\|}<0\right)$ at $z=0.5 R_{E}(r=$ $3.0 R_{E}$ radial distance) for the four simulations. Wave activity reaches $z=0.5 R_{E}$ at $t \sim 7 \mathrm{~s}$. In the lowest-amplitude case, there is little electron acceleration; the differential electron flux shows some modulation, but no enhancements. In simulation $B$, we see small enhancements in the $\sim 100 \mathrm{~s} \mathrm{eV}$ range. As expected, as the initial wave amplitude is increased, more electron acceleration can be seen in the differential electron energy flux, with enhancements at $\sim 1 \mathrm{keV}$ for simulation $\mathrm{C}$ and significant enhancements between 1 and $10 \mathrm{keV}$ for simulation D. In each simulation, there is temporal structure to the accelerated electron signatures for $5<t<8 \mathrm{~s}$, as tenuous energy-dispersed electron beams that have escaped the influence of the wave arrive ahead of the wave packet. After $\sim 8 \mathrm{~s}$, temporal structure is only seen in the low-amplitude simulations $\mathrm{A}$ and $\mathrm{B}$. The simulations with larger initial wave amplitudes show almost no coherent temporal structure to the accelerated electrons, which is a consequence of the destruction of the trapping islands above this location and the resulting overlap of accelerated electrons from different phases of the wave packet.

\section{Discussion}

[23] The motivation for our numerical investigations is the observational link between dispersive SAWs and aurora (see 

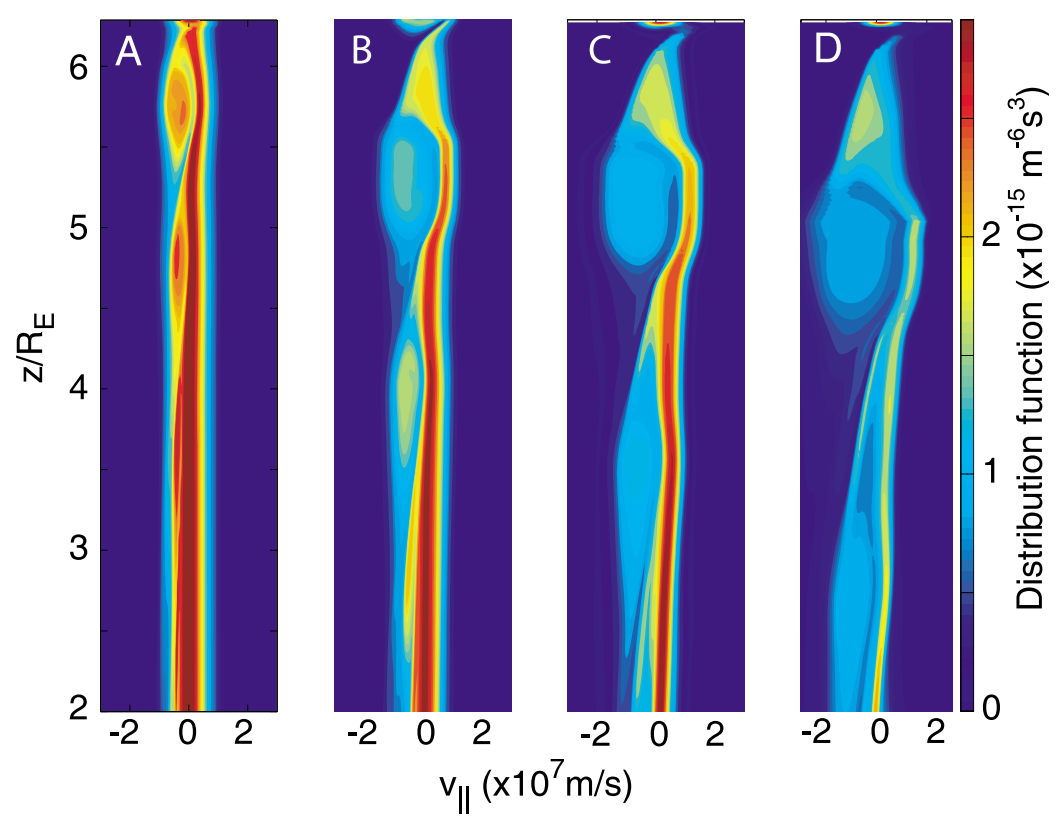

Figure 3. Snapshots of the distribution function for $\mu=0$ and $t \sim 7.8 \mathrm{~s}$ for the four simulations A, B, C, and D.

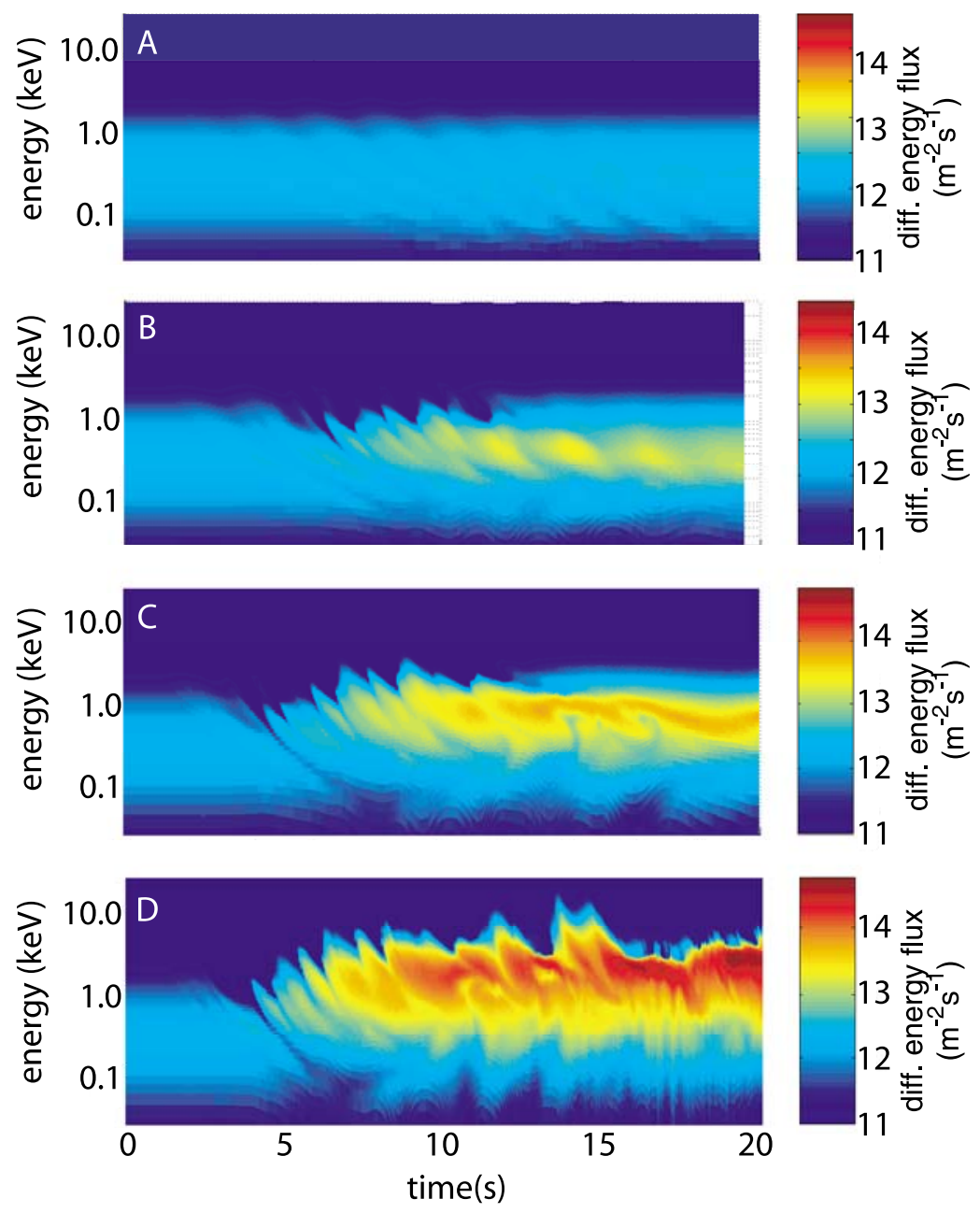

Figure 4. Differential electron energy flux $J_{E}$ for downward, field-aligned electrons at the point $z=0.5 R_{E}$ $\left(r \sim 3.0 R_{E}\right.$ radial distance) for each simulation $\mathrm{A}, \mathrm{B}, \mathrm{C}$, and $\mathrm{D}$. 


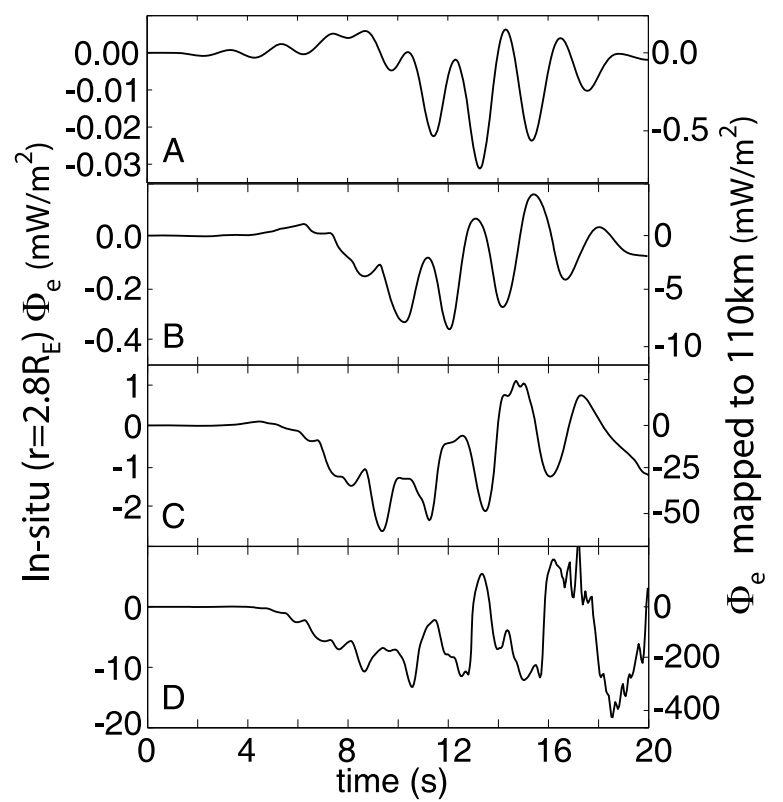

Figure 5. Parallel energy flux of accelerated particles determined at $z=0.25 R_{E}$ or $r \sim 2.8 R_{E}$ radial distance (left-hand axis) for each simulation. The right-hand axis indicates the amount of energy flux once it is mapped to $110 \mathrm{~km}$ altitude. Negative values indicate downward energy flux.

section 1 for a discussion of the observational evidence). The results from the simulations provided much insight into the physics of the shear Alfvén wave interaction with warm electrons, but to associate the resulting accelerated electrons with measurable auroral brightening, we must look at the resulting electron electron energy flux.

[24] In velocity coordinates, the electron energy flux is given by [Semeter et al., 2001]

$$
\Phi_{e}=\int_{-\infty}^{\infty} \int_{0}^{\infty} \frac{1}{2} m_{e}\left(v_{\|}^{2}+v_{\perp}^{2}\right) v_{\|} f_{e}\left(v_{\|}, v_{\perp}\right) 2 \pi v_{\perp} d v_{\perp} d v_{\|},
$$

while in simulation coordinates it is given by

$$
\begin{aligned}
\Phi_{e}= & \int_{0}^{\mu_{M}} \int_{-p_{M}}^{p_{M}} \frac{1}{2} B_{0}\left\{\left[p_{\|}-\frac{q_{e}}{m_{e}} A_{\|}(z)\right]^{2}+2 \mu \frac{B_{0}}{m_{e}}\right\}\left[p_{\|}-\frac{q_{e}}{m_{e}} A_{\|}(z)\right] \\
& \cdot f_{e}\left(z, p_{\|}, \mu\right) d p_{\|} d \mu,
\end{aligned}
$$

where the factor of $2 \pi$ is included in the normalization of $f_{e}\left(z, p_{\|}, \mu\right)$. Figure 5 shows the electron energy flux at $z \sim$ $0.25 R_{E}\left(r \sim 2.8 R_{E}\right)$ in each simulation $\mathrm{A}, \mathrm{B}, \mathrm{C}$, and D. Negative values indicate downward flux toward the ionosphere. Note that the simulation $\Phi_{e}$ are calculated at a radial distance much farther from the Earth than observations of the electron energy flux associated with auroral arcs, which are usually obtained from rocket measurements $(\sim 300-1000 \mathrm{~km}$ altitude) or from the FAST satellite (450-4200 km altitude). In the absence of simulations that would extend to lower altitudes (too computationally expensive to run in practice), we use the simulation value of precipitating electron energy flux at $r \sim 2.8 R_{E}$ to estimate the electron energy flux that impinges on the upper atmosphere as a result of acceleration by SAWs in the warm magnetosphere.
[25] As the accelerated electrons travel toward the ionosphere and into a converging magnetic field, it is expected that the electron energy flux should increase. If we estimate the electron energy flux at the ionosphere using a similar mapping procedure as for the wave Poynting vector in Figure $2 \mathrm{~d}$, then we obtain the values indicated on the righthand axes of Figure 5. However, there are a number of assumptions inherent in this choice of mapping:

[26] 1. All accelerated electrons are exactly field-aligned, and none are decelerated by the mirror force as the electron beam travels toward the ionosphere, thereby decreasing their contribution to the electron energy flux. Figure 6 shows the distribution function as a function of parallel and perpendicular velocity for simulation $\mathrm{C}$ at $z=2.8 R_{E}$ and $t=7 \mathrm{~s}$. The accelerated electrons can be clearly seen in the bottom half of the figure with negative parallel velocity (toward the ionosphere). The dashed white lines indicate the loss cone at this location in the dipolar magnetic field. Electrons above these lines mirror and become trapped in the magnetosphere, whereas electrons below these lines precipitate into the ionosphere. Some of these precipitating electrons lose parallel velocity because of the mirror force. It is clear from the distribution function that the biggest contributions to the parallel energy flux come from the electrons that are most field aligned, and that the number of electrons that would be affected by the mirror force is small, so this assumption is reasonable.

[27] 2. The energy flux shown in Figure 5 is caused by precipitating beam electrons, and there is no contribution to the energy flux from the parallel current owing to the wave. It was shown before [Watt et al., 2005] that the electrons that carry the SAW parallel current are locally accelerated and then decelerated and are unlikely to precipitate to cause the aurora. Because the energy flux is calculated from a moment of the full distribution function [equation (6)], it is difficult to separate the energy flux caused by the precipitating electrons from the energy flux caused by the SAW parallel current. The

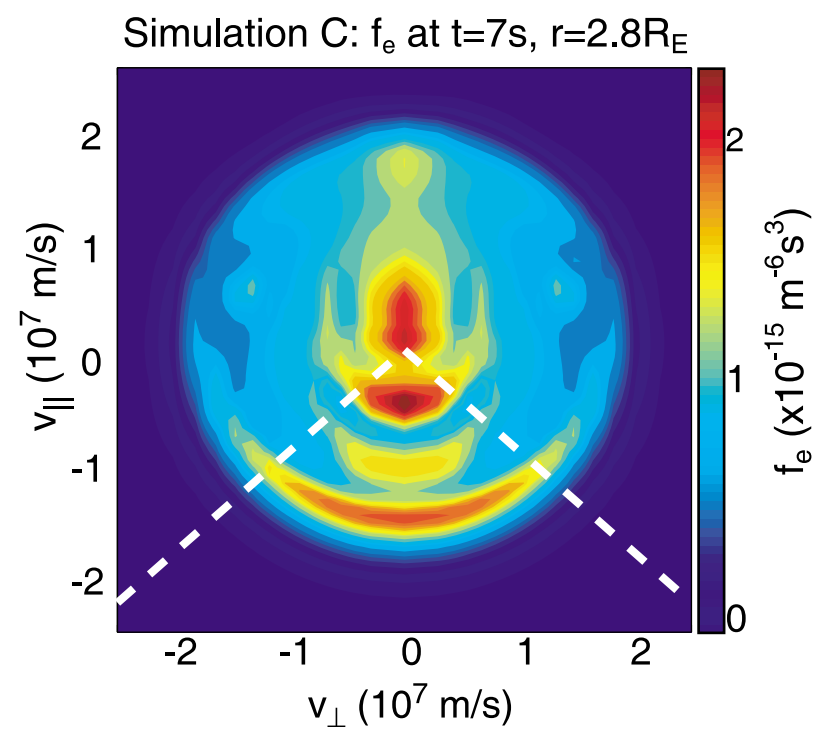

Figure 6. Snapshot of the distribution function at $r=2.8 R_{E}$ and $t=7 \mathrm{~s}$ from simulation C. Dashed white lines indicate the loss cone at this location. 


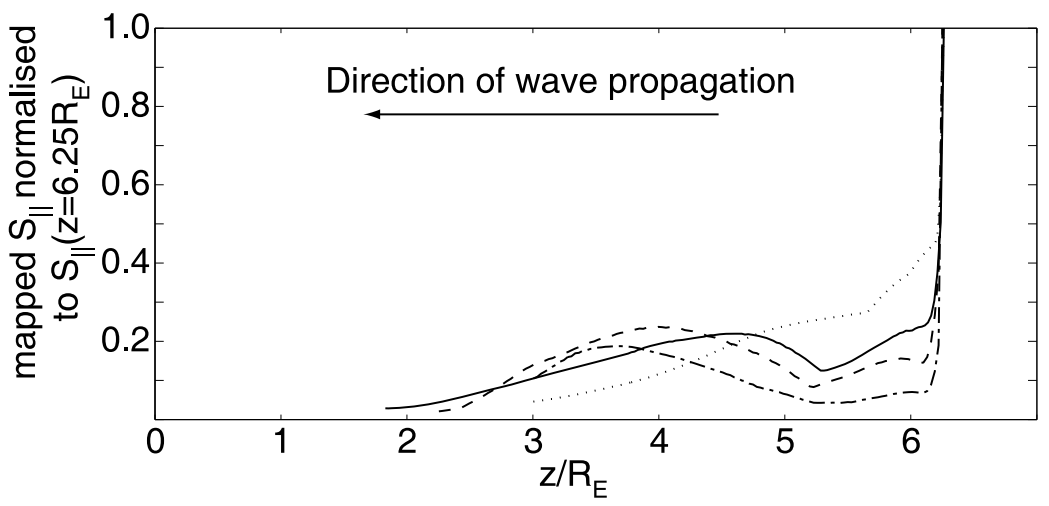

Figure 7. Maximum downward field-aligned Poynting flux at each point in the upper regions of the simulation domain, mapped to $110 \mathrm{~km}$ altitude. Results from each simulation are normalized to the mapped Poynting flux at $z=6.25 R_{E}$.

large oscillations in $\Phi_{e}$ for $t>8 \mathrm{~s}$ in Figure 5 have a period of $T \sim 2 \mathrm{~s}$ and are probably caused by the sloshing motion of the background electrons to carry the transient SAW current perturbations. This contribution to the energy flux is both downward and upward, whereas the contribution from the precipitating electrons is downward, and the separation of these effects is difficult. If we compare the size of $\Phi_{e}$ at $t \sim 7 \mathrm{~s}$ (just before the waves reach $r=2.8 R_{E}$ ) with the approximate amplitude of the $T \sim 2 \mathrm{~s}$ perturbations in $\Phi_{e}$, then they appear roughly similar, suggesting that the contribution to the energy flux from the current-carrying electrons is about the same as the contribution from the precipitating electrons.

[28] 3. There is no further acceleration of the electrons between this point and auroral emission altitudes. The wave parallel electric field in the simulations becomes greatly diminished before reaching the lower boundary of the simulation. However, there may be further acceleration owing to reflection and steepening of the wave at $\sim 1 R_{E}$ altitude where the Alfvén speed peaks [Lysak and Song, 2003a, 2003b; Chaston, 2006]. These effects are beyond the scope of the studies reported in this paper, although future simulations with extended simulation domains should be able to study all possible acceleration regions along auroral field lines.

[29] The first two assumptions imply that the simple mapping procedure overestimates the energy flux incident on the ionosphere (by at most a factor of 2), whereas the last assumption implies that it is underestimated.

[30] As discussed by Hallinan et al. [2001] and StenbaekNielsen et al. [1998], auroral brightness is more readily determined from linear plots of energy flux than from an electron spectrogram, such as those presented in Figure 4. The threshold for visible auroral brightening is $\Phi_{e}>1 \mathrm{~mW} / \mathrm{m}^{2}$ [e.g., Stenbaek-Nielsen et al., 1998]. The estimates obtained using the aforementioned considerations indicate that the wave modeled in simulation A generates insufficient energy flux to create visible aurora. However, the waves modeled in simulations B, C, and D do create sufficient precipitating electron flux to create visible auroral displays. Studies of electron acceleration caused by SAWs in uniform plasma [Watt et al., 2005; Watt and Rankin, 2002, 2008b] show that the energy and number of electrons that are accelerated through a single interaction with a SAW pulse depend sensitively on the wave amplitude, and these simulations in nonuniform plasma confirm that the electron energy flux owing to SAW acceleration is very sensitive to initial wave amplitude.

[31] The parameters used in these simulations were chosen with guidance from observations. As discussed in section 3, the initial wave amplitudes for simulations $\mathrm{B}$ and $\mathrm{C}\left(E_{\perp} \sim 20\right.$ $45 \mathrm{mV} / \mathrm{m}$ ) are comparable to many of the observations presented in the recent review by Keiling [2009]. Should we assume, however, that spacecraft observations of SAWs represent times before or after the waves have transferred energy to the electrons? Figure 7 shows the maximum instantaneous downward field-aligned Poynting flux at each grid cell in all four simulations mapped to ionospheric altitudes $(\sim 110 \mathrm{~km})$. Every effort was made to distinguish the Poynting flux owing to the original downward propagating wave from the interference generated when the wave reflects at the lower boundary, so results are not shown for lower regions of the simulation domain $\left(z<2 R_{E}\right)$. The mapped Poynting flux from each simulation was normalized to the value of mapped Poynting flux at the top end of the simulation, demonstrating that, for each simulation in this study, the shear Alfvén waves immediately lose $55-90 \%$ of their Poynting flux as they enter the simulation domain. This energy loss occurs in about $0.1-0.15 R_{E}$, or $4-5$ grid points. After the immediate transfer of wave energy flux to electron energy flux, the wave slowly loses more energy to the electrons, over a distance of $\sim 1 R_{E}$. As the wave continues to move through the plasma, this trend is momentarily reversed (except in simulation $\mathrm{A}$ ), with the wave gaining energy from the electrons (for $4.6<z<5.3 R_{E}$ in simulation B; $4<z<$ $5.2 R_{E}$ in simulation $\mathrm{C}$, and $3.7<z<5.2 R_{E}$ in simulation D). In simulation $A$, the wave does not gain energy from the electrons, but the rate at which wave energy is lost to the electrons is greatly reduced. In each of simulations B-D, the region over which the wave gains energy back from the electrons is where the trapping islands (see Figure 3) can be seen. Below this region, the wave parallel electric field diminishes, electrons are no longer trapped between successive wave crests, and the electrons can escape the influence of the wave, taking the wave energy flux with them as they travel toward the lower boundary. We can conclude that the majority of energy transfer between waves and particles occurs in a very short time and over a very small distance. Because observations [Wygant et al., 2002] show the presence of both waves and accelerated electrons at the same time 
and place, the wave amplitudes from in situ measurements are most likely to be an indication of the size of the waves after some of the wave energy was lost to the electrons. The initial wave amplitude used in simulation $\mathrm{D}$ is therefore both appropriate and realistic, because observations would be more likely to measure net wave amplitude once the electrons are trapped in the wave.

[32] It is not yet clear how magnetospheric SAWs develop the short perpendicular scales required to accelerate auroral electrons. It does however seem more likely that they would evolve through some nonlinear wave-wave process in a broadband wave disturbance [see, e.g., Wygant et al., 2002; Chaston et al., 2008] rather than being generated at a point source with a single well-defined wave number, as modeled here. However, the results from these simulations demonstrate that SAWs can be effective in the acceleration of auroral electrons at distances $>4 R_{E}$ radial distance. In fact, these idealized results demonstrate the exact mechanism by which shear Alfvén waves can interact readily with electrons in warm plasma and transfer the majority of their downward Poynting flux to field-aligned electron energy flux, as suggested by Keiling et al. [2002, 2003]. Future modeling of this wave-particle interaction should include the generation mechanism of these waves and the evolution of the waves and wave-particle interactions in the direction perpendicular to the magnetic field. The results reported in this paper demonstrate that the region $>4 R_{E}$ from the Earth is very important for auroral electron acceleration by shear Alfvén waves.

\section{Conclusions}

[33] Using self-consistent kinetic simulations of shear Alfvén waves in a nonuniform magnetic field, we investigated in detail the acceleration of electrons caused by a shear Alfvén wave of short perpendicular extent which is introduced into warm magnetospheric plasma at a radial distance of $r \sim 8 R_{E}$. As first reported by Watt and Rankin [2009], SAWs in plasma with $v_{\text {th,e }} / v_{A}>1$ undergo nonlinear damping caused by electron trapping in the parallel electric field of the wave. In this work, we show how the energy flux and characteristic energy of precipitating electrons varies with initial wave amplitude.

[34] The energy flux of the precipitating electrons is calculated at the lower boundary of the simulation domain, at a radial distance of $2.8 R_{E}$ for four simulations with different initial wave amplitudes. By estimating how this energy flux could be mapped to the ionosphere, assuming that it scales with the converging magnetic field and that most of the accelerated electrons are very field aligned and do not lose much parallel velocity because of the mirror force, the energy flux of the precipitating electrons is predicted to excite detectable aurora in three cases, given a detection threshold of $\sim 1 \mathrm{~mW} / \mathrm{m}^{2}$. The energy of accelerated electrons can reach $1-$ $10 \mathrm{keV}$ for wave and plasma parameters comparable to previously reported in situ observations. Thus, magnetospheric SAWs at radial distances $>4 R_{E}$ from the Earth can directly accelerate electrons with sufficient energy flux to explain auroral brightenings seen from the ground and in space. Wave-particle interactions in this region will be investigated in future work to determine the range of wave parameters conducive to the process.
[35] Acknowledgments. This work was supported by the Canadian Space Agency (CSA) and the Natural Sciences and Engineering Research Council of Canada (NSERC). C.E.J.W. would like to acknowledge support from Research Support in AICT at the University of Alberta during simulation code development.

[36] Robert Lysak thanks Christopher Chaston and John Wygant for their assistance in evaluating this manuscript.

\section{References}

Andersson, L., N. Ivchenko, J. Clemmons, A. A. Namgaladze, B. Gustavsson, J. E. Wahlund, L. Eliasson, and R. Y. Yurik (2002a), Electron signatures and Alfvén waves, J. Geophys. Res., 107(A9), 1244, doi:10.1029/ 2001JA900096.

Andersson, L., J. E. Wahlund, J. Clemmons, B. Gustavsson, and L. Eliasson (2002b), Electromagnetic waves and bursty electron acceleration: Implications from Freja, Ann. Geophys., 20(2), 139-150.

Angelopoulos, V., J. A. Chapman, F. S. Mozer, J. D. Scudder, C. T. Russell, K. Tsuruda, T. Mukai, T. J. Hughes, and K. Yumoto (2002), Plasma sheet electromagnetic power generation and its dissipation along auroral field lines, J. Geophys. Res., 107(A8), 1181, doi:10.1029/2001JA900136.

Angelopoulos, V., et al. (2008), Tail reconnection triggering substorm onset, Science, 321, 931-935, doi:10.1126/science.1160495.

Asamura, K., et al. (2009), Sheared flows and small-scale Alfvén wave generation in the auroral acceleration region, Geophys. Res. Lett., 36, L05105, doi:10.1029/2008GL036803.

Baumjohann, W. (1993), The near-Earth plasma sheet: An AMPTE/IRM perspective, Space Sci. Rev., 64, 141-163.

Boehm, M. H., C. W. Carlson, J. P. Mcfadden, J. H. Clemmons, and F. S. Mozer (1990), High-resolution sounding rocket observations of largeamplitude Alfvén waves, J. Geophys. Res., 95(A8), 12,157-12,171.

Chaston, C. C. (2006), ULF waves and auroral electrons, in Magnetospheric ULF waves: Synthesis and new directions, edited by K. Takahashi, P. J. Chi, R. E. Denton, and R. L. Lysak, p. 239, AGU, Washington, D. C.

Chaston, C. C., C. W. Carlson, R. E. Ergun, and J. P. McFadden (2000), Alfvén waves, density cavities and electron acceleration observed from the FAST spacecraft, Phys. Scr., T84, 64-68.

Chaston, C. C., J. W. Bonnell, L. M. Peticolas, C. W. Carlson, J. P. McFadden, and R. E. Ergun (2002), Driven Alfvén waves and electron acceleration: A FAST case study, Geophys. Res. Lett., 29(11), 1535, doi:10.1029/2001GL013842.

Chaston, C. C., J. W. Bonnell, C. W. Carlson, J. P. McFadden, R. E. Ergun, and R. J. Strangeway (2003a), Properties of small-scale Alfvén waves and accelerated electrons from FAST, J. Geophys. Res., 108(A4), 8003, doi:10.1029/2002JA009420.

Chaston, C. C., L. M. Peticolas, J. W. Bonnell, C. W. Carlson, R. E. Ergun, J. P. McFadden, and R. J. Strangeway (2003b), Width and brightness of auroral arcs driven by inertial Alfvén waves, J. Geophys. Res., 108(A2), 1091, doi:10.1029/2001JA007537.

Chaston, C. C., et al. (2005), Energy deposition by Alfvén waves into the dayside auroral oval: Cluster and FAST observations, J. Geophys. Res., 110, A02211, doi:10.1029/2004JA010483.

Chaston, C. C., C. W. Carlson, J. P. McFadden, R. E. Ergun, and R. J. Strangeway (2007), How important are dispersive Alfvén waves for auroral particle acceleration?, Geophys. Res. Lett., 34, L07101, doi:10.1029/ 2006GL029144.

Chaston, C. C., C. Salem, J. W. Bonnell, C. W. Carlson, R. E. Ergun, R. J. Strangeway, and J. P. McFadden (2008), The turbulent Alfvénic aurora, Phys. Rev. Lett., 100, 175003.

Christon, S. P., D. G. Mitchell, D. J. Williams, L. A. Frank, C. Y. Huang, and T. E. Eastman (1988), Energy-spectra of plasma sheet ions and electrons from approximately $50 \mathrm{eV} / e$ to approximately $1 \mathrm{MeV}$ during plasma temperature transitions, J. Geophys. Res., 93(A4), 2562-2572.

Clark, A. E., and C. E. Seyler (1999), Electron beam formation by smallscale oblique inertial Alfvén waves, J. Geophys. Res., 104(A8), 17,23317,249 .

Damiano, P. A., and A. N. Wright (2005), Two-dimensional hybrid MHDkinetic electron simulations of an Alfvén wave pulse, J. Geophys. Res., 110(A1), A01201, doi:10.1029/2004JA010603.

Damiano, P. A., R. D. Sydora, and J. C. Samson (2003), Hybrid magnetohydrodynamic-kinetic model of standing shear Alfvén waves, J. Plasma Phys., 69, 277-304

Damiano, P. A., A. N. Wright, R. D. Sydora, and J. C. Samson (2007), Energy dissipation via electron energization in standing shear Alfvén waves, Phys. Plasmas, 14(6), 062904.

Dombeck, J., C. Cattell, J. R. Wygant, A. Keiling, and J. Scudder (2005), Alfvén waves and Poynting flux observed simultaneously by Polar and 
FAST in the plasma sheet boundary layer, J. Geophys. Res., 110 A12S90, doi:10.1029/2005JA011269.

Ergun, R. E., L. Andersson, Y. J. Su, D. L. Newman, M. V. Goldman W. Lotko, C. C. Chaston, and C. W. Carlson (2005), Localized paralle electric fields associated with inertial Alfvén waves, Phys. Plasmas, 12(7), 072901

Genot, V., P. Louarn, and F. Mottez (2000), Electron acceleration by Alfvén waves in density cavities, J. Geophys. Res., 105(A12), 27,61127,620 .

Genot, V., F. Mottez, and P. Louarn (2001), Particle acceleration linked to Alfvén wave propagation on small scale density gradients, Phys. Chem. Earth Part C, 26(1-3), 219-222.

Genot, V., P. Louarn, and F. Mottez (2004), Alfvén wave interaction with inhomogeneous plasmas: Acceleration and energy cascade towards small-scales, Ann. Geophys., 22(6), 2081-2096.

Goertz, C. K., and R. W. Boswell (1979), Magnetosphere-ionosphere coupling, J. Geophys. Res., 84(A12), 7239-7246.

Hallinan, T. J., J. Kimball, H. C. Stenbaek-Nielsen, K. Lynch, R. Arnoldy, J. Bonnell, and P. Kintner (2001), Relation between optical emissions, particles, electric fields, and Alfvén waves in a multiple rayed arc, J. Geophys. Res, 106(A8), 15,445-15,454.

Horne, R. B., and M. P. Freeman (2001), A new code for electrostatic simulation by numerical integration of the Vlasov and Ampère equations using MacCormack's method, J. Comput. Phys., 171(1), 182-200.

Ivchenko, N., G. Marklund, K. Lynch, D. Pietrowski, R. Torbert, F. Primdahl, and A. Ranta (1999), Quasiperiodic oscillations observed at the edge of an auroral arc by Auroral Turbulence 2, Geophys. Res. Lett, 26(22), 3365-3368

Janhunen, P., A. Olsson, C. T. Russell, and H. Laakso (2006), Alfvénic electron acceleration in aurora occurs in global Alfvén resonosphere region, Space Sci. Rev., 122(1-4), 89-95, doi:10.1007/s11214-0067017-5.

Keiling, A. (2009), Alfvén waves and their roles in the dynamics of the Earth's magnetotail: A review, Space Sci. Rev., 142(1-4), 73-156, doi: $10.1007 / \mathrm{s} 11214-008-9463-8$.

Keiling, A., J. R. Wygant, C. Cattell, W. Peria, G. Parks, M. Temerin, F. S Mozer, C. T. Russell, and C. A. Kletzing (2002), Correlation of Alfvén wave Poynting flux in the plasma sheet at 4-7 $R_{E}$ with ionospheric electron energy flux, J. Geophys. Res., 107(A7), 1132, doi:10.1029/ 2001JA900140.

Keiling, A., J. R. Wygant, C. A. Cattell, F. S. Mozer, and C. T. Russell (2003), The global morphology of wave Poynting flux: Powering the aurora, Science, 299(5605), 383-386.

Kletzing, C. A. (1994), Electron acceleration by kinetic Alfvén waves, J. Geophys. Res., 99(A6), 11,095-11,103.

Kletzing, C. A., and S. Hu (2001), Alfvén wave generated electron time dispersion, Geophys. Res. Lett., 28(4), 693-696.

Kletzing, C. A., J. D. Scudder, E. E. Dors, and C. Curto (2003), Auroral source region: Plasma properties of the high-latitude plasma sheet, J. Geophys. Res., 108(A10), 1360, doi:10.1029/2002JA009678.

Leveque, R. J. (2002), Finite Volume Methods for Hyperbolic Problems, Cambridge Texts Appl. Math., 1st ed., 558 pp., Cambridge Univ. Press, New York.

Lynch, K. A., D. Pietrowski, R. B. Torbert, N. Ivchenko, G. Marklund, and F. Primdahl (1999), Multiple-point electron measurements in a nightside auroral arc: Auroral Turbulence II particle observations, Geophys. Res. Lett., 26(22), 3361-3364.

Lysak, R. L., and W. Lotko (1996), On the kinetic dispersion relation for shear Alfvén waves, J. Geophys. Res., 101(A3), 5085-5094.

Lysak, R. L., and Y. Song (2003a), Nonlocal kinetic theory of Alfvén waves on dipolar field lines, J. Geophys. Res., 108(A8), 1327, doi:10.1029/2003JA009859

Lysak, R. L., and Y. Song (2003b), Kinetic theory of the Alfvén wave acceleration of auroral electrons, J. Geophys. Res., 108(A4), 8005, doi:10.1029/2002JA009406.

Lysak, R. L., and Y. Song (2005), Nonlocal interactions between electrons and Alfvén waves on auroral field lines, J. Geophys. Res., 110, A10S06, doi:10.1029/2004JA010803

Morooka, M., et al. (2004), Cluster observations of ULF waves with pulsating electron beams above the high latitude dusk-side auroral region, Geophys. Res. Lett., 31, L05804, doi:10.1029/2003GL017714.

Nakamura, T. K. (2000), Parallel electric field of a mirror kinetic Alfvén wave, J. Geophys. Res., 105(A5), 10,729-10,737.

Olsson, A., and P. Janhunen (1998), Field-aligned conductance values estimated from Maxwellian and kappa distributions in quiet and disturbed events using Freja electron data, Ann. Geophys. Ser. A, 16(3), 298-302.

Østgaard, N., K. Snekvik, A. L. Borg, A. Åsnes, A. Pedersen, M. Øieroset, T. Phan, and S. E. Haaland (2009), Can magnetotail reconnection pro- duce the auroral intensities observed in the conjugate ionosphere?, J. Geophys. Res., 114, A06204, doi:10.1029/2009JA014185.

Rae, I. J., et al. (2009), Near-Earth initiation of a terrestrial substorm, J. Geophys. Res., 114, A07220, doi:10.1029/2008JA013771.

Rankin, R., and V. T. Tikhonchuk (1998), Numerical simulations and simplified models of nonlinear electron inertial Alfvén waves, J. Geophys. Res., 103(A9), 20,419-20,433.

Rankin, R., J. C. Samson, and V. T. Tikhonchuk (1999a), Parallel electric fields in dispersive shear Alfvén waves in the dipolar magnetosphere, Geophys. Res. Lett., 26(24), 3601-3604.

Rankin, R., J. C. Samson, and V. T. Tikhonchuk (1999b), Discrete auroral arcs and nonlinear dispersive field line resonances, Geophys. Res. Lett. 26(6), 663-666.

Semeter, J., and E. M. Blixt (2006), Evidence for Alfvén wave dispersion identified in high-resolution auroral imagery, Geophys. Res. Lett., 33(13), L13106, doi:10.1029/2006GL026274.

Semeter, J., D. Lummerzheim, and G. Haerendel (2001), Simultaneous multispectral imaging of the discrete aurora, J. Atmos. Sol. Terr. Phys. 63(18), 1981-1992.

Semeter, J., C. J. Heinselman, G. G. Sivjee, H. U. Frey, and J. W. Bonnell (2005), Ionospheric response to wave-accelerated electrons at the poleward auroral boundary, J. Geophys. Res., 110, A11310, doi:10.1029/ 2005JA011226.

Semeter, J., M. Zettergren, M. Diaz, and S. Mende (2008), Wave dispersion and the discrete aurora: New constraints derived from high-speed imagery, J. Geophys. Res., 113, A12208, doi:10.1029/2008JA013122.

Seyler, C. E., and K. Liu (2007), Particle energization by oblique inertial Alfvén waves in the auroral region, J. Geophys. Res., 112, A09302, doi:10.1029/2007JA012412.

Stenbaek-Nielsen, H. C., T. J. Hallinan, D. L. Osborne, J. Kimball, C. Chaston, J. McFadden, G. Delory, M. Temerin, and C. W. Carlson (1998), Aircraft observations conjugate to FAST: Auroral arc thicknesses, Geophys. Res. Lett., 25(12), 2073-2076.

Streltsov, A. V., and W. Lotko (1999), Small-scale, "electrostatic" auroral structures and Alfvén waves, J. Geophys. Res., 104(A3), 4411-4426.

Su, Y. J., S. T. Jones, R. E. Ergun, and S. E. Parker (2004), Modeling of field-aligned electron bursts by dispersive Alfvén waves in the dayside auroral region, J. Geophys. Res., 109, A11201, doi:10.1029/ 2003JA010344.

Su, Y.-J., R. E. Ergun, S. T. Jones, R. J. Strangeway, C. C. Chaston, S. E. Parker, and J. L. Horwitz (2007), Generation of short-burst radiation through Alfvénic acceleration of auroral electrons, J. Geophys. Res., 112, A06209, doi:10.1029/2006JA012131.

Summers, D., and R. M. Thorne (1992), A new tool for analyzing microinstabilities in space plasmas modeled by a generalized Lorentzian (kappa) distribution, J. Geophys. Res., 97(A11), 16,827-16,832.

Swift, D. W. (2007), Simulation of auroral electron acceleration by inertial Alfvén waves, J. Geophys. Res., 112, A12207, doi:10.1029/2007JA012423.

Sydorenko, D., R. Rankin, and K. Kabin (2008), Nonlinear effects in the inoosphere Alfvén resonator, J. Geophys. Res., 113, A10206, doi:10.1029/2008JA013579.

Thompson, B. J., and R. L. Lysak (1996), Electron acceleration by inertial Alfvén waves, J. Geophys. Res., 101(A3), 5359-5369.

Tikhonchuk, V. T., and R. Rankin (2000), Electron kinetic effects in standing shear Alfvén waves in the dipolar magnetosphere, Phys. Plasmas, 7 , 2630

Tikhonchuk, V. T., and R. Rankin (2002), Parallel potential driven by a kinetic Alfvén wave on geomagnetic field lines, J. Geophys. Res., 107(A7), 1104, doi:10.1029/2001JA000231.

Tsiklauri, D., J. I. Sakai, and S. Saito (2005), Particle-in-cell simulations of circularly polarised Alfvén wave phase mixing: A new mechanism for electron acceleration in collisionless plasmas, Astron. Astrophys. 435(3), 1105-1113

Vaivads, A., et al. (2003), What high altitude observations tell us about the auroral acceleration: A cluster/DMSP conjunction, Geophys. Res. Lett., 30(3), 1106, doi:10.1029/2002GL016006.

van Leer, B. (1974), Towards ultimate conservative difference scheme. 2. Monotonicity and conservation combined in a second-order scheme J. Comput. Phys., 14(4), 361-370.

Watt, C. E. J., and R. Rankin (2002), Electron acceleration due to inertial Alfvén waves in a non-Maxwellian plasma, J. Geophys. Res., 107(A9), 1244, doi:10.1029/2001JA900096.

Watt, C. E. J., and R. Rankin (2008a), DK-1D: A drift-kinetic simulation tool for modelling the shear Alfvén wave and its interaction with collisionless plasma, Plasma Phys. Controlled Fusion, 50(7), 074008.

Watt, C. E. J., and R. Rankin (2008b), Electron acceleration and parallel electric fields due to kinetic Alfvén waves in plasma with similar thermal and Alfvén speeds, Adv. Space Res., 42(5), 964-969. 
Watt, C. E. J., and R. Rankin (2009), Electron trapping in shear Alfvén waves that power the aurora, Phys. Rev. Lett., 102(4), 045002.

Watt, C. E. J., R. Rankin, and R. Marchand (2004), Kinetic simulations of electron response to shear Alfvén waves in magnetospheric plasmas, Phys. Plasmas, 11(4), 1277-1284.

Watt, C. E. J., R. Rankin, I. J. Rae, and D. M. Wright (2005), Self-consistent electron acceleration due to inertial Alfvén wave pulses, J. Geophys. Res., 110, A10S07, doi:10.1029/2004JA010877.

Watt, C. E. J., R. Rankin, I. J. Rae, and D. M. Wright (2006), Inertial Alfvén waves and acceleration of electrons in nonuniform magnetic fields, Geophys. Res. Lett., 33, L02106, doi:10.1029/2005GL024779.
Wygant, J. R., et al. (2000), Polar spacecraft based comparisons of intense electric fields and Poynting flux near and within the plasma sheettail lobe boundary to UVI images: An energy source for the aurora, J. Geophys. Res., 105(A8), 18,675-18,692.

Wygant, J. R., et al. (2002), Evidence for kinetic Alfvén waves and parallel electron energization at 4-6 $R_{E}$ altitudes in the plasma sheet boundary layer, J. Geophys. Res., 107(A8), 1201, doi:10.1029/2001JA900113.

R. Rankin and C. E. J. Watt, Department of Physics, University of Alberta, Edmonton, AB T6G 2G7, Canada. (rankin@phys.ualberta.ca; cwatt@phys.ualberta.ca) 